\title{
Pesquisa e metapesquisa sobre comunicação na América Latina
}

\section{Research and meta-research on}

\section{communication in Latin America}

\author{
RA Ú L F ENTES NAVARROa \\ Universidad de Guadalajara, Departamento de Estudios de la Comunicación Social. Guadalajara - Jalisco, México \\ Universidad Jesuita de Guadalajara (Iteso), Departamento de Estudios Socioculturales. Guadalajara - Jalisco, \\ México
}

\section{RESUMO}

Este artigo aborda e propõe a discussão, a partir de uma perspectiva comunicacional, das relações estratégicas e práticas entre a pesquisa e a metapesquisa da comunicação na América Latina. Para tanto, ressalta-se a importância da história como fator complementar essencial à estatística, bem como algumas reflexões controversas sobre postulados que parecem indispensáveis para retomar o debate na comunidade acadêmica e perante os órgãos responsáveis pela avaliação e orientação da ciência.

Palavras-chave: Metapesquisa, pesquisa sobre comunicação, história

\begin{abstract}
This paper argues and proposes for discussion, from a communicational perspective, the strategic and practical relations between research and the meta-research of communication in Latin America. To this end, the importance of history as an essential complementary factor to statistics is emphasized, as well as some controversial reflections on postulates that seem indispensable to re-discuss in the academic community and before the bodies responsible for the evaluation and orientation of science.
\end{abstract}

Keywords: Meta-research, communication research, history 


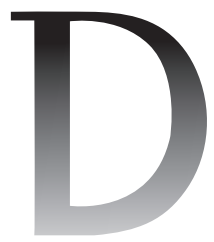

${ }^{1}$ No original: "cuatro décadas de internacionalización académica en el campo de estudios de la comunicación en Latinoamérica”.

${ }^{2}$ No original: "to improve the quality of scientific studies in biomedicine and beyond".

${ }^{3}$ No original: "its own methods, reporting, reproducibility, evaluation, and incentives".
STE ENSAIO PRETENDE expor e incitar a discussão sobre alguns resultados de um exercício acadêmico que o autor teve muitas oportunidades de desenvolver e aproveitar durante a maior parte de sua carreira, e que nos anos mais recentes permitiu-lhe propor reflexões comemorativas críticas, no marco de quatro décadas de diversas organizações acadêmicas com as quais esteve envolvido na maior parte desse tempo: o Conselho Nacional para o Ensino e a Pesquisa em das Ciências da Comunicação (Coneicc), no México, constituído em 1976; a Sociedade Brasileira de Estudos Interdisciplinares da Comunicação (Intercom), criada em 1977; a Associação Latino-Americana de Investigadores da Comunicação (Alaic), fundada em 1978 e reconstruída em 1989; a Associação Mexicana de Investigadores da Comunicação (Amic), fundada em 1979. Revisitar, com o objetivo de historiar, "quatro décadas de internacionalização acadêmica no campo de estudos da comunicação na América Latina"' (Fuentes, 2016), é uma proposta de pesquisa que enfoca a recuperação das interpretações encontradas, defendidas e publicadas desde os anos de 1980 até a atualidade, dentro e fora da região. Nesse sentido, é um exercício de metapesquisa, e segue o exemplo reflexivo do que outros autores denominam "metanálise" em sentido estatístico (Levine \& Hullet, 2015); "metadiscurso" nos contextos da Retórica e Pragmática (Craig, 2015b); "análises crítico-reflexivas sobre as práticas de investigação e dos estudos na área" como Epistemologia da Comunicação (Lopes, 2016); ou "metaconhecimento" em comunicação, seja produzido por meio de métodos históricos ou estatísticos (Günther \& Domahidi, 2017).

\section{A METAPESQUISA, RECURSO CIENTÍFICO CONTEMPORÂNEO}

De acordo com John Ioannidis (2018), codiretor do Metrics (Meta-Research Innovation Center at Stanford - Centro de Inovação de Metapesquisa da Universidade Stanford - que busca "reforçar a qualidade dos estudos científicos em biomedicina e mais além"2), a metapesquisa é o estudo da própria investigação: "seus métodos, informes, reprodutibilidade, avaliação e incentivos"3 (p. 1). Essas cinco áreas constitutivas da metapesquisa correspondem, respectivamente, a indagações sobre a concepção e a realização dos estudos, sua disseminação e comunicação pública, sua verificação e autocorreção, a valorização de sua qualidade e padrões científicos e a concessão de suas recompensas e sanções. As práticas institucionalizadas da pesquisa científica e o crescimento exponencial de seus produtos podem ser analisados cada vez mais facilmente com evidências empíricas e métodos de larga escala, avaliando sua efetividade com recursos cada vez mais confiáveis, o que é uma necessidade crescente na complexa relação 
ciência-sociedade. O Manifesto por uma ciência reproduzivel parte da premissa de que "melhorar a confiabilidade e a eficácia da pesquisa científica irá aumentar a credibilidade da literatura científica publicada e acelerar a descoberta"4 (Munafò et al., 2017, p. 1), objetivos que supõe de interesse universal e que mostram grande continuidade com os aportes clássicos da cientometria, como a análise e os índices de citação na ciência (Garfield, 1955, 1972) e a "Big Science" de Price (1963). A reprodutibilidade da ciência é, sobretudo, um antídoto contra os plágios e as fraudes, que aumentaram, ou ao menos se tornaram mais visíveis, a partir do ápice das publicações open access e a tendência a reduzir toda avaliação da produção científica aos índices, um grave problema contemporâneo, que precisa ser compensado por critérios qualitativos, como propõe Gianfranco Pacchioni (2018), citando a "Lei de Goodhart: quando uma medida se converte em uma meta, deixa de ser uma boa medida" (p. 95). Em outras palavras, a estatística e a história, o quantitativo e o qualitativo, são mutuamente necessários em função do sentido da metapesquisa.

Enquanto isso, no site da Metrics ${ }^{6}$, evidencia-se uma continuidade metainvestigativa da metapesquisa, de acordo com as cinco áreas e seis regiões geográficas, com links para as referências e os estudos relatados: 8374, dos quais $75 \%$ vêm da América do Norte e Europa, e 75\% estão localizados nas áreas de Métodos e Relatos, o que obviamente significando que a metainvestigação também é heterogênea e fragmentária. Não há informações quantitativas tão claramente acessíveis sobre a participação de pesquisadores sociais ou humanistas neste projeto, institucionalizado apenas em 2014 na área biomédica. No entanto, presume-se que "dado os tipos de perguntas dirigidas, a metapesquisa interage com muitas outras disciplinas"' (Ioannidis et al., 2015, p. 3), incluindo história, filosofia, psicologia e sociologia da ciência; estatística, ciência da informação, ciência da computação, cienciometria e bibliometria; investigações organizacionais e operacionais, ética, ciências da comunicação, políticas públicas e economia comportamental.

Para os profissionais das ciências da comunicação de qualquer lugar do mundo é bem conhecido que o processo de identificar os limites de qualquer disciplina, para não mencionar um campo densamente multidisciplinar, é "dinâmico e um tanto arbitrário, exigindo atualizações e aperfeiçoamentos constantes" (Ioannidis et al., 2015, p. 4). Mas há, sem dúvida, outras implicações que devem ser consideradas, especialmente quando se trabalha em uma região relativamente marginal em termos de produção científica, como a América Latina (Fuentes, 2018d). Assim, este artigo sobre a pesquisa e a metapesquisa acadêmicas da comunicação nesse subcontinente exige uma revisão dos aperfeiçoamentos alcançados nos padrões científicos dessa especialidade do "metaconhecimento"
${ }^{4}$ No original: "Improving the reliability and efficiency of scientific research will increase the credibility of the published scientific literature and accelerate discovery".

${ }^{5}$ No original: "when a measure becomes a target, it ceases to be a good measure. This is known today as Goodhart's law".

${ }^{6}$ Recuperado de https:// metrics.stanford.edu/mapvisualization

${ }^{7}$ No original: "Given the types of questions addressed, metaresearch interfaces with many other established disciplines".

${ }^{8}$ No original: "a dynamic and somewhat arbitrary pro-cess, which requires continuous updates and refinements". 


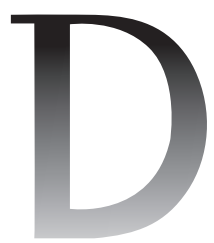

${ }^{9}$ No original: "the examination of how teaching programmes as well as research activity have helped to institutionalize the area as one with a discrete, if muchdebated, academic identity".

${ }^{10}$ No original: "make us more sharply aware of the various and sometimes precarious modes of institutionalization by which study of public communication has developed, breaking out of older professional and academic frameworks while continuing selectively to draw upon them".

${ }^{11}$ No original: "persistencia de la teoría negada”.

(Evans \& Foster, 2011) ou da "ciência da ciência" (Fortunato et al., 2018), e uma atualização da produção concreta, a partir da escala básica dos dados, em contextos socioculturais de graus relativamente menores de consolidação e reconhecimento, mas, justamente por isso, não menos relevantes.

Pode-se observar, já de saída, que as questões fundamentais que a metapesquisa nos permite abordar de forma cada vez mais analítica e responder de maneiras cada vez mais convincentes estão presentes em toda a história das ciências da comunicação, especialmente em relação à sua identidade teórica (Pasquali, 1978; Verón, 1987; Martín-Barbero, 1987; Craig, 1999; Curran \& Park, 2000; Martín-Serrano, 2007; Nordenstreng, 2007; Moragas, 2011; Sodré, 2014; Vizer \& Vidales, 2016). Além disso, o interesse pela busca de padrões transnacionais e internacionais na institucionalização do estudo da comunicação tem gerado uma grande proliferação de abordagens de metapesquisa nos últimos anos (Koivisto \& Thomas, 2008; Park \& Pooley, 2008; Gehrke \& Keith, 2015; Simonson \& Park, 2016; Averbeck-Lietz, 2017).

Estas e outras contribuições para a metapesquisa de comunicação foram sistematicamente revistas por John Corner (2019) em um artigo recente, destacando, em particular, a expansão do trabalho histórico sobre o estudo da comunicação para incluir contextos internacionais e "o exame de como os programas de ensino e a atividade de pesquisa contribuíram para institucionalizar a área com uma identidade acadêmica discreta, embora muito debatida"' (p. 1). Os três fatores destacados por Corner (2019) são os diversos contextos nos quais os programas se originaram, os vínculos históricos da pesquisa com as práticas profissionais e o impacto das novas mídias na história recente do ensino e da pesquisa. A metapesquisa contribui, finalmente, para "tornar-nos mais conscientes dos diversos, e por vezes precários, modos de institucionalização por meio dos quais se desenvolveu o estudo da comunicação pública, rompendo com as estruturas acadêmicas e profissionais mais antigas, ao mesmo tempo em que recorrendo a elas"10 (p. 9). A última parte desta avaliação relembra inevitavelmente aquela "persistência da teoria negada" ${ }^{11}$ que Jesús Martín-Barbero (1982, p. 101) denunciou na América Latina há muitos anos.

$\mathrm{Na}$ literatura especializada atual, de fato, parece evidenciar-se uma condição que Elihu Katz resumiu em 2013, durante uma reunião internacional de historiadores do estudo da comunicação, dizendo que "o nosso problema (metapesquisa) é não sabermos qual é o nosso problema (pesquisa)”. A diversidade de objetos de conhecimento que foram agrupados sob o termo comunicação e a diversidade de arranjos institucionais para organizar o seu estudo acadêmico em diferentes países e regiões se tornaram o foco para seções de história de associações acadêmicas internacionais (como a Associação Internacional de Comunicação, ICA, e a Associação Internacional para Pesquisa em Mídia e 
Comunicação, IAMCR). Embora "até agora, a maioria das histórias terem sido nacionais, com foco predominante na América do Norte e Europa Ocidental"12 (Simonson \& Peters, 2008, p. 764), tem se reforçado recentemente uma perspectiva que "nos ajuda a ver como o estudo organizado de comunicação ao mesmo tempo tem refletido, refratado e promovido a geopolítica transnacional, padrões institucionais de educação e profissionalização e as formas de conhecer e agir"13 (Simonson \& Park, 2016, p. 2) determinantes da vida coletiva. Nesta linha, ganhou forte impulso a busca por marcos sócio-históricos adequados para apoiar uma investigação transnacional dos processos de formação do campo acadêmico da comunicação (Loblich \& Scheu, 2011; Craig, 2015a; Loblich \& Averbeck-Lietz, 2016). E a mesma indagação pode ser percebida dentro da América Latina e sobre ela (Marques de Melo, 1992, 1998, 2007; Fuentes, 1992, 1998, 2006; Orozco, 1997; Bolaño, Cimadevilla, \& Crovi 2015; Crovi, 2018; Crovi \& Cimadevilla, 2018; Crovi \& Trejo, 2018).

\section{BASES E SENTIDO DA METAPESQUISA DA COMUNICAÇÃO NA AMÉRICA LATINA}

De acordo com Silvio Waisbord (2014), na América Latina "o campo de estudos de comunicação/mídia permanece unido, ainda que fragmentado"14 (p. 2), porque, ao contrário do que aconteceu nos Estados Unidos ou na Europa Ocidental, surgiu a partir de uma trajetória intelectual comum e seu "cânone" é mais homogêneo intelectualmente, enquanto estudos de comunicação, incorporando "textos clássicos da tradição do imperialismo cultural e midiático, a semiótica estrutural e a análise do discurso, a filosofia continental, os estudos culturais e a economia política"15 (p. 7). Neste subcontinente, "o campo está aberto às tendências intelectuais e de desenvolvimento globais e regionais"16 (p. 7), coincidindo com o julgamento de Miquel de Moragas (2011): "a pesquisa em comunicação na América Latina não é homogênea, mas se baseia em algo muito especial: compartilhar a diversidade e desconstruir os aparatos teóricos sobre a comunicação baseados na experiência das grandes metrópoles do mundo ocidental desenvolvido"17 (p. 302). No livro citado, Moragas exibe uma atualização bem documentada do que, trinta anos atrás, ele já enfatizara: "na América Latina, devido à vivacidade das mudanças sociais e transformações comunicativas, as implicações políticas de pesquisa em comunicação surgiram mais claramente do que em qualquer outro contexto"18. Mas agora, embora essa atividade "esteja plenamente incorporada na história das conquistas e dificuldades dos processos de luta contra a ditadura, a pobreza e a dominação" ${ }^{19}$, com o passar dos anos, superando as dificuldades, "também irá libertando-se
${ }^{12}$ No original: "To this point, most histories have been national, with the bulk of attention devoted to North America and Western Europe".

${ }^{13}$ No original: "gets us closer to seeing how organized communication study has at once reflected, refracted, and advanced transnational geopolitics, institutional patterns of education and professionalization, and ways of knowing and acting".

${ }^{14}$ No original: "the field of communication/media studies remains united yet fragmented".

${ }^{15}$ No original: "It features classic texts of the media/ cultural imperialism tradition, structural semiotics and discourse analysis, continental philosophy, cultural studies, and political economy".

${ }^{16}$ No original: "the field has been open to global and regional development and intellectual trends".

${ }^{17}$ No original: "la investigación de la comunicación en América Latina no es homogénea, pero se basa en algo muy particular: compartir la diversidad y deconstruir los aparatos teóricos sobre comunicación basados en la experiencia ajena de las grandes metrópolis del mundo occidental desarrollado".

${ }^{18}$ No original: “en Latinoamérica, por la viveza del cambio social y las transformaciones comunicativas, aparecieron más claramente que en ningún otro contexto las implicaciones políticas de la investigación sobre la comunicación”.

${ }^{19}$ No original: "se encarna, plenamente, en la historia de los logros y las dificultades de los procesos de lucha contra la dictadura, la pobreza y la dominación". 


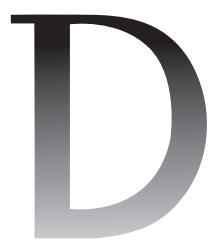

${ }^{20}$ No original: "también irá liberándose de las influencias teóricas dominantes, construyendo su propia intertextualidad teórica, discutiendo, renovando, descartando teorías".

${ }^{21}$ No original: "In this heterogeneous field, what is needed is not a pressure for conformity but the production of more and better connections among different lines of work".

${ }^{22}$ No original: "asking the big questions that connect different lines of work it is a matter that far exceeds the domain of theory".

${ }^{23}$ No original: "a cosmopolitan view that places theoretical questions at the center of the analysis, cautiously and critically approaches theories produced in different settings, and is engaged with debates in the global community of communication and media scholars".

das influências teóricas dominantes, construindo sua própria intertextualidade teórica, discutindo, renovando, descartando teorias"20 (p. 178).

Nesse mesmo ano, em um congresso internacional de pesquisadores de comunicação, Craig Calhoun (2011), então presidente do Conselho de Pesquisa em Ciências Sociais (Social Science Research Council - SSRC), afirmou que "neste campo heterogêneo, o que é necessário não é pressão pela conformidade, mas produzir mais e melhores conexões entre diferentes linhas de trabalho" 21 (p. 1495), para o que a teoria tem um papel especial, embora "fazer as grandes perguntas que conectam diferentes linhas de trabalho é algo que vá muito além da esfera da teoria"22 (p. 1495). E, evidentemente, na América Latina há uma grande experiência na concepção e enfrentamento dos desafios da "realidade social" múltipla e desigual, como também reconhece Waisbord (2014), para quem o necessário é adotar uma abordagem analítica semelhante ao que já gerou avanços inovadores no passado, "uma visão cosmopolita que coloca as questões teóricas no núcleo da análise, abordagens teóricas produzidas em diferentes locais, assumidos de maneira cautelosa e crítica e comprometidos com os debates da comunidade acadêmica global"23 (p. 2).

A este respeito, deve ser reiterado que a história do pensamento e da ação estratégica em comunicação são dois aspectos inseparáveis de um projeto acadêmico ainda para se desenvolver plenamente como uma tarefa de pesquisa, integrando-se como interpretação e convicção críticas, a ser discutido e compartilhado dentro e fora da academia, dentro e fora da região latino-americana (Fuentes, 2018a, pp. 304-305). Nesse projeto de metapesquisa, como tem sido assumido há muito tempo (Fuentes, 1999, 2006), uma recuperação histórica da pesquisa latino-americana sobre comunicação e das perspectivas para enfrentar os desafios das transformações globais hoje em andamento requer o desenvolvimento de ações coletivas de vários tipos e níveis, começando com um aspecto básico de infraestrutura de pesquisa e metapesquisa: sistemas e serviços de documentação.

A estrutura geral desses sistemas foi estabelecida em 1968, pela Rede Internacional de Centros de Documentação sobre Pesquisa e Políticas de Comunicação (Network of Documentation Centers on Communication Research and Policies - Comnet), cuja coordenação foi assumida pela Unesco em 1971, com o objetivo de melhorar e acelerar as trocas regionais e internacionais de informação e documentação, buscando contribuir significativamente para o desenvolvimento científico no campo da comunicação em todas as regiões do mundo (Pisarek, 1982, pp. 1-2). Oito redes regionais foram estabelecidas: África, Estados Árabes, Ásia, Pacífico, Caribe, América Latina, América do Norte e Europa, que derivaram diferentemente da crise da Unesco nos anos de 1980. 
Porém, embora no campo latino-americano de pesquisa em comunicação os sistemas de documentação acadêmica tenham sido frequentemente reconhecidos como importantes, poucos foram ampliados e desenvolvidos suficientemente, especialmente na transição para a digitalização e para o open access, seguindo acordos tais como a Declaração de Berlim (convocada pela Sociedade Max Planck e aprovada em 22 de outubro de 2003 por representantes de várias instituições europeias ${ }^{24}$ ): "é necessário apoiar novas possibilidades de difusão de conhecimento, não só pela via clássica, mas também pelo paradigma do acesso aberto através da internet”25 (MPG, 2003).

O desenvolvimento de plataformas tecnológicas tem, obviamente, criado, em poucos anos e de uma forma muito extensa, horizontes de possibilidades quase inimagináveis nas décadas de 1970 e 1980 para cumprir as metas de democratização do conhecimento e da comunicação da ciência e da cultura. Mas os projetos latino-americanos que conseguiram tirar proveito dos recursos da internet para fortalecer a consecução dos objetivos fundamentais da documentação e divulgação acadêmica são infelizmente escassos. Um exemplo representativo, mas não excepcional, nessa perspectiva de escassez pode ser uma antologia de textos críticos sobre La Investigación en Comunicación Social en América Latina 1970-2000, editado e reeditado em Lima como um livro impresso (Portugal, 2012). O volume reúne 35 trabalhos, a maioria deles de acesso difícil e independente, mas de referência muito útil e talvez indispensável no conjunto para caracterizar os debates e orientações teóricas e políticas sobre a comunicação ao longo de três décadas na América Latina, mas não foi editado digitalmente nem está disponível como tal na internet. Por outro lado, exemplos positivos incluem o Portcom, um repositório institucional de produção científica, a memória da Intercom ${ }^{26}$ no Brasil, e o $c c$-doc, que oferece acesso sistematizado a referências - e, em muitos casos, aos documentos completos - de pesquisa acadêmica sobre a comunicação no México ${ }^{27}$.

\section{FUNDAÇÕES DA METAPESQUISA DE COMUNICAÇÃO LATINO- -AMERICANA}

Em setembro de 1973, foi realizado na Costa Rica o primeiro "Seminário de Pesquisa em Comunicação na América Latina”, organizado pelo Centro Internacional de Estudos Superiores de Jornalismo para a América Latina (Ciespal). A importância fundamental desta reunião é geralmente aceita, pois foi a primeira vez que especialistas de diferentes países foram convidados a discutir o estado da arte no campo e para acordar um programa de ação com base em definições normativas gerais:

\author{
${ }^{24}$ Recuperado de http://mpg. \\ de/index.html \\ ${ }^{25}$ No original: "New \\ possibilities of knowledge \\ dissemination not only through \\ the classical form but also \\ and increasingly through the \\ open access paradigm via the \\ Internet have to be supported".
}

\footnotetext{
${ }^{26}$ Recuperado de http:// portcom.intercom.org.br/ portcom.php

${ }^{27}$ Recuperado de http://ccdoc. iteso.mx
} 


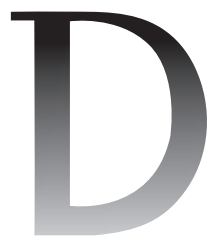

${ }^{28}$ No original: "El objetivo central de la investigación debe ser el análisis crítico del papel de la comunicación en todos los niveles de funcionamiento, sin omitir sus relaciones con la dominación interna y la dependencia externa y el estudio de nuevos canales, medios, mensajes, situaciones de comunicación, etc., que contribuyan al proceso de transformación social".

\footnotetext{
${ }^{29}$ No original: "La teoría de la comunicación y la metodología de la investigación elaboradas en los centros metropolitanos no siempre corresponden a la realidad y a las necesidades de investigación de los países atrasados y dependientes, no obstante lo cual se aplican, indiscriminadamente, a las situaciones de la región, con resultados obviamente inadecuados y a veces distorsionantes. Su uso ha sido inducido bajo el supuesto de que la teoría social es universal y de que su validez desborda el marco de los espacios culturales y de los procesos históricos".
} em todos os níveis de funcionamento, sem omitir suas relações com a dominação interna e dependência externa e o estudo de novos canais, meios, mensagens, situações de comunicação, etc., que contribuem para o processo de transformação social $^{28}$. (Ciespal, 1974, p. 15)

A inserção social da pesquisa em comunicação, como projeto de prática científica e exploração sistemática de suas contribuições para o desenvolvimento é, assim, desde o início, uma constante discursiva, que tem, ao mesmo tempo, a intenção de convocar e organizar, a partir de vários espaços institucionais, a produção e reprodução dos agentes de campo e de seus saberes (Beltrán, 2005). É também um objeto de disputa, eixo de convergência e divergência de interpretações, contribuições, recursos e articulações (Fuentes 2006, pp. 183-189). Embora tenha sido considerado "provisório e incompleto" pelos participantes do Seminário, o quadro conceitual supracitado não deixou dúvidas quanto à postura epistemológica e política adotada:

A teoria da comunicação e a metodologia de pesquisa desenvolvidas nos centros metropolitanos nem sempre correspondem à realidade e às necessidades de pesquisa dos países atrasados e dependentes, no entanto, são aplicadas, indiscriminadamente, às situações da região, com resultados obviamente inadequados e, por vezes, distorcidos. Seu uso tem sido induzido sob o pressuposto de que a teoria social é universal e que sua validade vai além da fronteira dos espaços culturais e dos processos históricos ${ }^{29}$. (Ciespal, 1974, p. 13)

Também foram discutidas "diretrizes gerais sobre metodologia” e uma avaliação das pesquisas sobre a comunicação na América Latina. O relatório final desse seminário, bem como alguns documentos trabalhados nele, especialmente aqueles produzidos para avaliar o desenvolvimento obtido pela a investigação até o momento na América Latina, são um ponto de partida especialmente interessante não só para rever quais tendências temáticas e teórico-metodológicas a pesquisa de comunicação tinha nas décadas transcorridas até então, mas também para analisar as mudanças nas condições socioculturais em que tais práticas se desenvolveram, com todas as disparidades, nos países latino-americanos, tanto nas dimensões de sua institucionalização quanto em seus fundamentos e orientações. A revisão e a interpretação crítica da documentação disponível eram indispensáveis, ainda que se levassem a reconhecer, nas palavras de Jorge Merino Utreras (1974), do Departamento de Pesquisa do Ciespal, que "nos oferecem um quadro sombrio do estado da investigação em nosso continente 
e a necessidade urgente de planejar o ensino e aplicação prática de teorias de pesquisa"30 (pp. 84-87). Até então, o Ciespal tinha conseguido recuperar para o seu Centro de Documentação 733 textos, dos quais 112 eram estritamente produtos de pesquisa "de campo e de laboratório" provenientes de treze países (pp. 88-103).

Um ano após o Seminário de Costa Rica, em setembro de 1974, Luis Ramiro Beltrán apresentou no Congresso da IAMCR em Leipzig seu famoso relato sobre "A pesquisa de comunicação na América Latina, investigação com antolhos?”. Com base na documentação compilada pela Ciespal, Beltrán (1974) listou as principais áreas de concentração temática, destacou as tendências relacionadas aos tópicos pesquisados e aos resultados obtidos nos últimos quinze anos, e observou que:

É óbvio que a pesquisa em comunicação na América Latina seguiu as orientações conceituais e metodológicas estabelecidas por pesquisadores na Europa e nos Estados Unidos. O significado disso, em essência, é que alguns estudos têm enfatizado a compreensão conceitual mais do que a produção de evidências empíricas, enquanto que outros estudos têm feito exatamente o contrário ${ }^{31}$. (p. 23)

Beltrán (1974) observou que "se os estudos existentes são classificados em descritivos, explicativos e preditivos, a maioria, provavelmente, estaria dentro da primeira categoria, alguns na segunda e menos na terceira" ${ }^{32}$ (p. 25). Embora a pesquisa de comunicação da América Latina tenha se alterado substancialmente em muitas dimensões e aspectos, não é possível dizer que tenha mudado muito em sua estrutura fundamental, especialmente em sua abordagem teórica e metodológica. Pelo contrário, assim como a produção do campo em todo o mundo, diversificou-se e fragmentou-se em todas as suas dimensões. Mas esse documento de Beltrán terminava significativamente com comentários sobre a "mitologia de uma ciência livre de valores"33 (p. 37) e sobre o "risco do dogmatismo" ${ }^{4}$ (p. 38). A oposição, de muitas formas, maniqueísta, entre o rigor da ciência e o compromisso político de transformação social, relacionada diretamente à polêmica travada pouco antes entre os grupos de pesquisadores liderados por Eliseo Verón, na Argentina, e Armand Mattelart, no Chile, dava lugar a uma pergunta crucial, com a qual Beltrán (1974) concluía seu relato: "Isso poderia significar que a pesquisa de comunicação latino-americana corre o risco de um dia substituir o funcionalismo ideologicamente conservador e metodologicamente rigoroso por um radicalismo sem rigor?”35 (p. 40).

O legado metodológico de Beltrán para o campo é inestimável em termos do afã de documentar, de maneira sistemática e detalhada, as várias posições
${ }^{30}$ No original: "nos da un panorama desalentador del estado de la investigación en nuestro continente y la apremiante necesidad de planificar la enseñanza y aplicación práctica de las teorías de la investigación".

${ }^{31}$ No original: "It is obvious that communication research in Latin America has followed conceptual and methodological orientations established by researchers in Europe and the United States. The effect of this, in essence, has meant that some studies have emphasized conceptual insight over empirically drawn data whereas other studies have done exactly the opposite".

${ }^{32}$ No original: "if the existing studies were to be classified into descriptive, explicative and predictive research categories, probably most would fall in the first category, some in the second and the least in the third".

${ }^{33}$ No original: "On the Mythology of a Value-Free Science".

${ }^{34}$ No original: "On the Danger of Dogmatism”.

${ }^{35}$ No original: "Could this mean that Latin American communication research may one day run into danger of substituting ideologically conservative and methodologically rigorous functionalism for unrigorous radicalism?". 
e contribuições que têm orientado as práticas de pesquisa acadêmica sobre a comunicação na América Latina em referência a seus vetores utópicos e suas articulações com três dimensões centrais de sua orientação social: desenvolvimento, dependência e democracia. Nessa linha, é necessário reconhecer os esforços para enriquecer o autorreconhecimento crítico do campo, em contextos diferentes e com diferentes formatos, incluindo as publicações formais, ainda que parciais e fragmentárias. Entre esses esforços, sem dúvida, se destacam os realizados por um grande número de pesquisadores brasileiros, latino-americanos e de muitas outras nacionalidades, em resposta às iniciativas incansáveis de José Marques de Melo (1988, 1992, 1998, 2007), o qual, por várias décadas, liderou e documentou todo um movimento para combater "o relativo desconhecimento das novas gerações sobre o pensamento construído por nossos pioneiros" latino-americanos no campo. Seu legado também é inestimável.

Uma questão relevante é: ainda que em termos de pesquisa o movimento comunicacional mais intenso dos anos de 1960 e 1970 tenha ocorrido principalmente fora das faculdades, a institucionalização do campo acadêmico na América Latina aumentou consideravelmente nos anos de 1980 e 1990 com base em dois pilares fundamentais: um, os processos de identificação e integração continental, canalizados ou conduzidos por organizações acadêmicas internacionais, como a Associação latino-americana de investigadores da Comunicação (Alaic, fundada em 1978) e a Federação latino-americana de Faculdades de Comunicação Social (Felafacs, instituída em 1981) (Fuentes, 2016). O outro pilar dessa institucionalização é formado pelas revistas acadêmicas e científicas de vocação explicitamente latino-americana: destacadamente, a mais durável, Chasqui, editada pelo Ciespal, no Equador, desde 1972; a mítica Comunicación y Cultura, publicada sucessivamente no Chile, na Argentina e no México entre 1973 e 1985; Diálogos de la Comunicación, publicada no Peru e na Colômbia pela Felafacs desde 1987; e, mais recentemente, a Revista Latinoamericana de Ciencias de la Comunicación, editada pela Alaic no Brasil desde 2004. É também importante mencionar os produtos de uma iniciativa da Alaic, a qual promoveu, no início dos anos de 1980, o desenvolvimento e publicação de sistematizações documentais nacionais sobre a comunicação no Peru, Chile, Brasil, Colômbia, Argentina, México e Bolívia (Peirano \& Kudo, 1982; Munizaga \& Rivera, 1983; Marques de Melo, 1984; Anzola \& Cooper, 1985; Rivera, 1986; Fuentes, 1988; Beltrán et al., 1990). Essa iniciativa não teve, infelizmente, como notado acima, o seguimento desejado, apesar de valiosas exceções (Almengor et al., 1992; Aguirre, 1996; Kunsch \& Denker, 1997; Rivera, 1997; Herrera et al., 2000; Del Valle, 2004; Maldonado, 2014; Portillo, 2016; Torrico, 2016). 


\section{MEMÓRIA DOCUMENTADA E INSTITUCIONALIZAÇÃO ACADÊMICA}

$\mathrm{Na}$ atualidade é possível utilizar recursos acadêmico-científicos cada vez mais variados e precisos para o desenvolvimento da metapesquisa no campo de estudos da comunicação, seja em suas diferentes áreas ("métodos, relatos, reprodutibilidade, avaliação e incentivos", seguindo o modelo da Metrics: Ioannidis, 2018); seja para desenvolver seus mapas ("de projetos, grupos, linhas, objetos de estudo e métodos de investigação sobre as práticas sociais da Comunicação" ${ }^{36}$, de acordo com a proposta formal do MapCom na Espanha: Piñuel et al., 2013) ou para promover no e a partir do Brasil a autorreflexão sistemática ("sobre os princípios, fundamentos e procedimentos que orientam a prática de um conhecimento científico particular", segundo o Grupo de Pesquisa Campo Comunicacional e Interfaces: Mattos et al., 2018). Em suma, a metapesquisa "tornou-se nos últimos anos uma especialidade essencial para reconhecer os processos de institucionalização, profissionalização e legitimação" ${ }^{37}$ no campo acadêmico e para a "construção de mapas heurísticos"38 (Fuentes, 2007, p. 166) que facilitem aos agentes responsáveis por seu desenvolvimento a coordenação estratégica de suas práticas, mesmo sem ter se consolidado ou aproveitado suficientemente.

Como categorias analíticas para a análise empírica do conteúdo de produtos de pesquisa publicados é possível distinguir três conjuntos básicos: a) contribuições e reflexões teóricas e epistemológicas; b) metodologias e instrumentos de pesquisa e c) dimensões e processos do campo acadêmico. Os três conjuntos incluem uma diversidade própria da episteme contemporânea, expressa em diferentes modelos e terminologias nos contextos relevantes para a análise das práticas, mas que, afinal, convergem com bastante facilidade. Assim, por exemplo, Robert T. Craig (2008) propõe extrair "recursos retóricos para construir e legitimar disciplinas"39 (p. 8) a partir de contextos da "história intelectual, institucional e sociocultural"40 (p. 8), enquanto o modelo desenvolvido por Löblich e Scheu (2011) baseia suas "funções heurísticas" para se inter-relacionar, em diferentes níveis teóricos e empíricos, à análise de "acadêmicos individuais, as instituições acadêmicas e os campos não científicos da sociedade"41 (p. 16). Antes deles, Fuentes (1998) havia assumido, como recurso heurístico metainvestigativo da comunicação no México, que a constituição de um campo acadêmico envolve processos inter-relacionados de escalas "individual" (constituição dos sujeitos; formação/conformação do habitus; profissionalização), "institucional" (institucionalização social, institucionalização cognitiva, especialização científica) e no campo "sociocultural" (autorreprodução, legitimação social e práticas "assimilação/acomodação") (p. 73).

Seguindo estas pistas e outras confluências, torna-se cada vez mais claro que, para reconhecer e reconstruir a "memória e historicidade da pesquisa em
${ }^{36}$ No original: "de proyectos, grupos, líneas, objetos de estudio y métodos de investigación sobre prácticas sociales de Comunicación".

${ }^{37}$ No original: "se ha transformado en los últimos años en una especialidad esencial para reconocer los procesos de institucionalización, profesionalización y legitimación".

${ }^{38}$ No original: "construcción de mapas heurísticos".

${ }^{39}$ No original: "Rhetorical resources for constructing and legitimizing disciplines".

${ }^{40}$ No original: "intellectual, institutional, and sociocultural history".

${ }^{41}$ No original: "individual scholars, scholarly institutions, and nonscientific fields of society". 


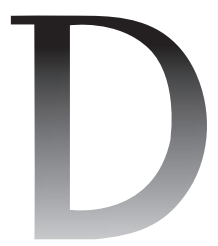

${ }^{42}$ No original: "continuously in the process of making, remaking, and unmaking itself".

${ }^{43}$ No original: un "sistema de efectos de sentido discursivos". comunicação na América Latina" (título eloquente e comprometido dado à conferência inaugural do $40^{\circ}$ Congresso Brasileiro de Ciências da Comunicação, o Intercom, realizada em Curitiba em 2017), o foco de atenção sobre as tensões e antagonismos pode ser muito mais esclarecedor dos processos de institucionalização que a imaginação de fluxos unidirecionais de influência ou recursos, ou a defesa de excepcionalismos históricos, nacionais ou regionais. Além disso, para contextualizar adequada e diversamente os processos de transnacionalização do campo não é possível separar uma reconstrução somente epistemológica da evolução das ideias, ou apenas uma série de reconhecimento dos méritos e contribuições de indivíduos, por mais extraordinários que tenham sido, ou apenas a explicação determinista, por fatores macroeconômicos ou geopolíticos das formas específicas de reprodução cultural ou, inclusive, ideológica de noções reducionistas de comunicação.

O desenvolvimento de um relato histórico consistente e orientador precisa reconhecer a complexidade e multiplicidade dos fatores envolvidos, dos processos histórico-sociais entrelaçados e não delimitados em cada uma das escalas espaço-temporais especificamente (Fuentes, 2018b). Como parte do mundo social "que se constrói, se desconstrói, continuamente se refaz"42 (Abbott, 2016, p. ix), o discurso científico-social implica uma autorrevisão permanente das suas premissas e de suas propostas, seus métodos e suas articulações com outras práticas e estruturas sociais, as quais, reflexivamente, toma como objeto. Em outras palavras, deve aproveitar e cultivar o desejo permanente que caracteriza o campo dos estudos da comunicação de questionar a si mesmo, de analisar e discutir suas fundações e fundamentos. Este aparente jogo de palavras, inspirado na semiótica social de Eliseo Verón (1987), é capaz de reconhecer e articular bem a dimensão histórica e social de um campo acadêmico num processo interminável de institucionalização, com a dimensão, intelectual ou epistemológica, de suas estruturas de produção e circulação de conhecimentos e métodos, suas teorias. Essa proposta, focada na concepção do conhecimento como "um sistema de efeitos de sentido discursivos"43 (p. 22), coloca a necessidade de uma teoria de fundações como "um processo sem fundador", uma vez que o próprio o processo de fundação tem a forma de uma rede intertextual capaz de se desdobrar em um determinado período temporal e, é claro, em um espaço específico, sob condições históricas concretas.

É discutível se esta premissa, ainda focada no discurso, necessariamente exclui os sujeitos localizados, enunciadores e enunciatários do discurso. Mas é possível defender, heurísticamente, que o estudo da comunicação teve certamente uma primeira fundação (nos Estados Unidos, imaginada e executada por Wilbur Schramm), mas também é possível reconhecer que houve e haverá várias outras 
(nos próprios Estados Unidos ou em outras regiões). Este ponto é central porque levanta a questão sobre o que são essas fundações e fundamentos diversos do estudo da comunicação? E as consequências de "a resposta à pergunta do porquê de o início não estar nos discursos: está contida nas condições de produção dos discursos sociais"44 (Verón, 1987, p. 29). Dessa forma, e dificilmente de outra, é possível defender a noção de uma teoria latino-americana de comunicação, cujos fundamentos e fundações podem ser aparentemente incompatíveis, dependente ou independentemente dos processos socioculturais derivados de uma fundação original, ideologicamente incapazes de serem reconhecidos como contingentes e excludentes (Fuentes, 2018c).

O Colóquio “Tecendo nossa história. Pesquisa da comunicação na América Latina”, organizado em 2016 pela Alaic e pela Unam na Cidade do México com "o objetivo de identificar elementos que permitam construir um relato regional do campo, sua organização, seus protagonistas e a investigação que está sendo conduzida” ${ }^{45}$ (Crovi \& Trejo, 2018, p. 8) certamente arejou as perspectivas prevalecentes sobre nossa história e ajudou a confirmar mais uma vez que, apesar das muitas diferenças que caracterizam as manifestações nacionais da institucionalização do estudo acadêmico comunicação, na América Latina existem vetores de identidade que há mais de meio século são referências de contraste e articulação tanto na própria escala regional quanto nos processos globais ou transnacionais dessa especialidade profissional e científica. Sem que se tenha literalmente proposto, a partir da experiência desse simpósio pode ser elaborada a hipótese de que o que prevalece hoje, tanto na comunicação social quanto em sua pesquisa acadêmica na América Latina, pode ser melhor entendido como uma tensão entre convergência e fragmentação, e não como uma estrutura polarizada (de oposição entre opções bem definidas), como a dinâmica desse campo foi percebida em períodos mais maniqueístas, e também, claro, como uma acumulação linear ao longo do tempo que nunca existiu (Fuentes, 2018b, p. 18).

\section{A INTERNACIONALIZAÇÃO DESINTEGRADA E O PENSAMENTO CRÍTICO LATINO-AMERICANO}

A caracterização hipotética da pesquisa em comunicação como sujeita a uma "internacionalização desintegrada" 46 (Fuentes, 2014), em tensão entre convergência e fragmentação, pode ter utilidade heurística em diferentes escalas. Assim, as quatro grandes fases temporais propostas por Simonson e Park (2016) em sua História internacional do estudo da comunicação, como o tempo em que se desenvolveram esses estudos em todo o mundo depois de uma longa "pré-história", são construídos a partir de três âmbitos: "sistemas educacionais e
${ }^{44}$ No original: "la respuesta a la cuestión del porqué del comienzo no se encuentra en los discursos: está contenida en las condiciones de producción de los discursos sociales".

${ }^{45}$ No original: "el propósito de identificar elementos que permitan construir un relato regional del campo, su organización, sus protagonistas y las investigaciones que se realizan".

${ }^{46}$ No original: "internacionalización desintegrada”. 


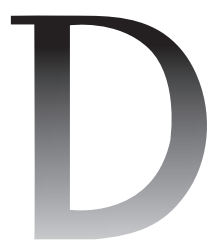

${ }^{47}$ No original: "pedagogy and educational systems", "practical application and other modes of reflective practice" e "research and theory".

${ }^{48}$ No original: "when dominant paradigms were challenged from multiple quarters issuing in ferments that fundamentally altered the communication study socially, politically, and intellectually".

pedagógicos", "aplicações práticas e outros modos de prática reflexiva" e "pesquisa e teorização"47 (p. 9). As quatro etapas vão, respectivamente, de 1870 a 1938, quando surge e se desenvolve um "espaço de problematização intelectual sobre a comunicação"; de 1939 a 1967, quando os processos de institucionalização desse campo acadêmico se consolidam; de 1968 a 1988, quando "os paradigmas dominantes foram confrontados a partir de múltiplos flancos, provocando fermentos que alteraram fundamentalmente o estudo da comunicação social, política e intelectualmente"48 (p. 9); e a partir de 1989, a etapa contemporânea no estudo da comunicação, caracterizada pela pluralização e disciplinarização.

Com algum atraso em certos momentos, e diferentes circunstâncias nacionais como contexto dos processos de institucionalização e legitimação dos estudos sobre a comunicação, assim como da consolidação de organizações e redes internacionais e transnacionais especializadas na América Latina, neste esquema cronológico podem ser localizados com alguma facilidade os principais marcos da história regional para reinterpretá-los, assim, em relação a contextos mais amplos do que os geralmente considerados e que transcendem as tradições, paradigmas ou correntes teóricas e epistemológicas como categorias ainda consideradas úteis por alguns autores latino-americanos. Por exemplo, durante a primeira fase (1870-1938) é possível reconhecer os estudos humanistas, históricos, literários e jurídicos, as quais, especialmente a propósito do jornalismo, mas também, de forma incipiente, do cinema e do rádio foram realizadas em vários países latino-americanos. No final deste período, as primeiras escolas (e uma cátedra) de jornalismo foram fundadas na América do Sul.

Durante o segundo período (1939-1967), em meio à tensão geopolítica e cultural gerada pela Segunda Guerra Mundial e a subsequente Guerra Fria, na América Latina as perspectivas funcionalistas e desenvolvimentistas de comunicação de massa confrontaram-se com as críticas e militantes derivadas do modelo socialista inspirado na Revolução Cubana. A fundação do Ciespal, em 1959, permitiu uma primeira referência transnacional para a disseminação de projetos de comunicação intra e extra-latino-americanos de alcance e orientação diversos. Além disso, em 1960 é criado no México o programa universitário de Ciências da Comunicação, de acordo com um projeto socioprofissional orientado pela filosofia e pelas humanidades, e em 1963 é publicado Comunicación y Cultura de Masas, de Antonio Pasquali, a primeira abordagem a uma síntese teórico-crítica escrita em espanhol.

Entre 1968 e 1988, durante a terceira fase, a pesquisa acadêmica da comunicação começou a se consolidar institucionalmente em vários países latino-americanos e se cruzaram processos sociopolíticos bastante intensos (golpes de Estado e retornos à democracia, guerras civis e repressão dos movimentos insurgentes, criação 
de blocos regionais, geopolíticos e comerciais), com debates, pressões e decisões governamentais, nem sempre consistentes, sobre o modelo de desenvolvimento adotado para os meios de difusão, especialmente o rádio e a televisão. Na época os estudos críticos sobre os fluxos de informação e de documentação transnacionais adquiriram relevância continental e global, assim como a correspondente denúncia do desequilíbrio e dependência dos países latino-americanos, coincidindo com movimentos semelhantes na África e nos países árabes (Simonson \& Park, 2016, p. 17). Essas questões foram fundamentais para a proposta de "políticas de comunicação nacional" e para o surgimento da "Nova Ordem Mundial de Informação e Comunicação”, defendida pela Unesco. Foram criados e se consolidaram os primeiros programas de pós-graduação (mestrado em 1972, doutorado em 1980, ambos no Brasil, Lopes, 2012, p. 32) e foram publicados vários livros fundacionais do pensamento crítico latino-americanos, como Conducta, estructura y comunicación, em 1968, e um ano depois Lenguaje y comunicación social, de Eliseo Verón; Pedagogia do oprimido e Extensão ou comunicação, de Paulo Freire; os Cuadernos da Realidad Nacional no Chile, entre 1969 e 1973, pelo grupo dirigido por Armand Mattelart; e, em 1987, De los medios a las mediaciones. Comunicación, cultura y hegemonía, de Jesús Martín Barbero.

Mas na década de 1990, na América Latina, assim como em outras regiões do mundo, a consolidação alcançada na institucionalização, na profissionalização e na legitimidade social do estudo da comunicação foi imediatamente contestada pelo surgimento de processos globais de grande impacto, precisamente, nos sistemas de comunicação. Simonson e Park (2016, p. 6) apontam como marcos determinantes as revoluções que começaram em 1989 na Europa Oriental e levaram à queda do comunismo, ao fim da Guerra Fria e à aceleração do neoliberalismo como fenômeno transnacional que modificador da configuração da mídia, da educação e tudo mais, começando com as tecnologias digitais, frequentemente vistas como fatores causais, talvez devido à amplitude de suas aplicações ou à eficácia de seu marketing. Nessa etapa contemporânea, as práticas de comunicação, as indústrias midiáticas e os imaginários sociais foram radicalmente transformados. Estes desenvolvimentos podem criar novos espaços nacionais e transnacionais de comunicação, aumentando a relevância social e profissional da educação no campo e reconfigurando tanto os temas de investigação quanto os meios para realizá-la (Simonson \& Park, 2016, p. 18) e para interpretá-la, é claro. Deve-se ressaltar que, dada a velocidade e a profundidade com que redes de mídia se transformam e convergem, com consequências divergentes, processos e estruturas até recentemente mantidos separados, como os da radiodifusão e das telecomunicações, ou do entretenimento e da educação, ou da política e da publicidade, ou de práticas públicas e privadas, parece clara a necessidade 


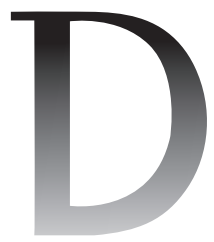

${ }^{49}$ No original: "it is a paradoxical effect of the consolidation of communication studies in the region that collaborative efforts aimed at producing distinct Latin American theory, and bringing it into dialogue with the work of other academic communities, have waned".

${ }^{50}$ No original: "beyond well-known structural factors such as the unequal access to resources (to attend conferences, acquire literature, outsource translation and editing services, etc.) or the expansion of English as 'universal' lingua franca".

${ }^{51}$ No original: "la teoría es uno de los espacios clave de la dependencia... pero la dependencia no consiste en asumir teorías producidas 'fuera'; lo dependiente es la concepción misma de la ciencia, del trabajo científico y su función en la sociedad.

Como en otros campos, también aquí lo grave no es que sean exógenos los productos, sino las estructuras mismas de producción". de outro esforço coletivo e sistemático de diagnóstico informado e de projeção compartilhada, de uma renovação crítica dos recursos da metapesquisa.

A partir daí, provavelmente, poderiam ser encontradas melhores formulações para o que Enghel e Becerra (2018) expressam, reconhecendo que "é um efeito paradoxal da consolidação dos estudos de comunicação na região a diminuição dos esforços de colaboração para produzir uma teoria propriamente latino-americana e colocá-la em diálogo com outras comunidades acadêmicas" 49 (pp. 116-117) e a necessidade de aperfeiçoar as perguntas sobre a circulação internacional da teoria da comunicação produzida na América Latina para ir "além dos fatores estruturais bem conhecidos, tais como o acesso desigual aos recursos (para assistir congressos, adquirir literatura, contratar traduções e serviços editoriais, etc.) ou a expansão do inglês como língua franca 'universal" "50 (p. 117). O que não parece suficientemente estabelecido na apresentação da edição especial de Communication Theory, coordenada por eles, é o modelo conceitual sobre a produção e circulação do conhecimento científico que subjaz aos muito valiosos e perspicazes, mas relativamente limitados, esforços para elaborar uma perspectiva mais crítica, sincrética e praxeológica sobre a comunicação científica, cada vez mais concentrada em um modo industrial (ou pós-industrial) de produção de bens e não necessariamente de sentido, uma tendência que, ao contrário do mundo desenvolvido, na América Latina não atingiu os mesmos níveis de consolidação hegemônica, apesar das pressões exercidas externamente e de dentro das próprias instituições científicas e educativas nacionais. Dado o reducionismo que prevalece mundialmente para avaliar a produção científica pelo fator de impacto dos periódicos, o acúmulo de citações e o Índice-h, deve-se recordar e rediscutir uma premissa formulada por Jesus Martín-Barbero em 1980:

a teoria é um dos espaços-chave da dependência... mas a dependência não consiste em assumir teorias produzidas "fora"; dependente é a própria concepção de ciência, de trabalho científico e de sua função na sociedade. Como em outros campos, também aqui o grave não é que os produtos sejam exógenos, mas sim as próprias estruturas de produção ${ }^{51}$. (Martín-Barbero, 1982, p. 101)

E as estruturas de produção cultural, os modelos de representação que subjazem às práticas são, provavelmente, a dimensão mais opaca para os próprios praticantes, os pesquisadores comprometidos com a acumulação de capital e a avaliação, observando certas regras e o investimento de determinados recursos, algumas e outros já definidos, se não em referência a outros fundamentos, talvez a outras fundações, a projetos de produção científica diferentes dos predominantes. $\mathbf{M}$ 


\section{REFERÊNCIAS}

Abbott, A. (2016). Processual sociology. Chicago e Londres: The University of Chicago Press.

Aguirre, J. M. (1996). De la práctica periodística a la investigación comunicacional. Hitos del pensamiento venezolano sobre comunicación social y cultura de masas. Caracas: Universidad Católica Andrés Bello.

Almengor, M. et al. (1992). La investigación en comunicación social en Panamá. Panamá: Instituto Nacional de Cultura; Editorial Mariano Arosemena.

Anzola, P. \& Cooper, P. (1985). La investigación en comunicación social en Colombia. Lima: Desco.

Averbeck-Lietz, S. (Ed.). (2017). Kommunikationswissenschaft im internationalen vergleich. Wiesbaden: Springer.

Beltrán, L. R. (1974). Communication research in Latin America: the blindfolded inquiry? Artigo apresentado na Conferência Científica Internacional Mass Communication and Social Consciousness in a Changing World. Leipzig: International Association for Mass Communication Research.

Beltrán, L. R. (2005). La comunicación para el desarrollo en Latinoamérica: Un recuento de medio siglo. Documento apresentado no Terceiro Congreso Panamericano de la Comunicación, Buenos Aires.

Beltrán, L. R., Suárez, C., \& Isaza. G. (1990). Bibliografía de Estudios sobre Comunicación en Bolivia. La Paz: Proinsa; IDRC.

Bolaño, C., Crovi, D., \& Cimadevilla, G. (Coords.). (2015). La contribución de América Latina al campo de la comunicación. Historia, enfoques teóricos, epistemológicos y tendencias de la investigación. Buenos Aires: Prometeo.

Calhoun, C. (2011). Communication as Social Science (and More). International Journal of Communication, 5(2011), 1479-1496.

Centro Internacional de Estudios Superiores de Periodismo (Comunicación) para América Latina. (1974). Seminario sobre la investigación de la comunicación en América Latina. Costa Rica 1973. Quito: Ciespal.

Centro Internacional de Estudios Superiores de Periodismo (Comunicación) para América Latina. (1977). Comunicación Social y Desarrollo. Compendios de Investigaciones sobre América Latina (2 Vols.). Quito: Ciespal.

Corner, J. (2019). Origins and transformations: histories of communication study. Media, Culture \& Society (2019), 1-11. doi: 10.1177/0163443718820666

Craig, R. T. (1999). Communication Theory as a Field. Communication Theory, 9(2), 119-161. doi: 10.1111/j.1468-2885.1999.tb00355.x

Craig, R. T. (2008). Communication in the conversation of disciplines. Russian Journal of Communication, 1(1), 7-23. doi: 10.1080/19409419.2008.10756694 
Craig, R. T. (2015a). The constitutive metamodel: a 16-year review. Communication Theory, 25(4), 356-374. doi: 10.1111/comt.12076

Craig, R. T. (2015b). Metadiscourse. In W. Donsbach (Ed.), The Concise Encyclopedia of Communication (pp. 386-387). Malden, MA: Wiley-Blackwell.

Crovi, D. (Coord.). (2018). Sociedad del conocimiento y comunicación. Reflexiones críticas desde América Latina. México: Alaic.

Crovi, D. \& Cimadevilla, G. (Coords.). (2018). Del mimeógrafo a las redes digitales. Narrativas, testimonios y análisis del campo comunicacional en el 40 aniversario de Alaic. México: Alaic.

Crovi, D. \& Trejo, R. (Coords.). (2018). Tejiendo nuestra historia. Investigación de la comunicación en América Latina. México: Unam.

Curran, J. \& Park M.-J. (Eds.). (2000). De-Westernizing Media Studies. Londres, UK; Nova York, NY: Routledge.

Del Valle Rojas, C. (2004). Metainvestigación de la comunicación en Chile. Tendencias y crítica. Temuco: Universidad de La Frontera.

Enghel, F. \& Becerra, M. (2018). Here and there: (re)situating Latin America in international communication theory, Communication Theory, 28(2), 111-130. doi: 10.1093/ct/qty005

Evans, J. A. \& Foster, J. G. (2011). Metaknowledge. Science, 331(6018), 721-725. doi: $10.1126 /$ science. 1201765

Fortunato, S. et al. (2018). Science of Science. Science, 359(6379), eaao0185. doi: $10.1126 /$ science.aao0185

Fuentes-Navarro, R (1988). La investigación de comunicación en México. Sistematización documental 1956-1986. México: Ediciones de Comunicación.

Fuentes-Navarro, R. (1992). Un campo cargado de futuro. El estudio de la comunicación en América Latina. México: Felafacs.

Fuentes-Navarro, R. (1998). La emergencia de un campo académico: Continuidad utópica y estructuración científica de la investigación de la comunicación en México. Guadalajara: Iteso; Universidad de Guadalajara.

Fuentes-Navarro, R. (1999). La investigación de la comunicación en América Latina: condiciones y perspectivas para el siglo XXI, Comunicación y Sociedad, (36), 105-132.

Fuentes-Navarro, R. (2006). Para documentar las relecturas y reescrituras del campo latinoamericano de investigación de la comunicación. In R. Fuentes-Navarro (Coord.), Instituciones y redes académicas para el estudio de la comunicación en América Latina (pp. 183-226). Guadalajara: Iteso.

Fuentes-Navarro, R. (2007). Fontes bibliográficas da pesquisa acadêmica nos cursos de Pós-Graduação em Comunicação no Brasil e no México: Uma 
aproximação da análise comparativa. MATRIZes, 1(1), 165-177. doi: 10.11606/issn.1982-8160.v1i1p165-177

Fuentes-Navarro, R. (2014). La investigación de la comunicación en América Latina: una internacionalización desintegrada. Oficios Terrestres, (31), 11-22. Fuentes-Navarro, R. (2016). Cuatro décadas de internacionalización académica en el campo de estudios de la comunicación en Latinoamérica. Anuario Electrónico de Estudios en Comunicación Social "Disertaciones", 9(2), 8-26. doi: 10.12804/disertaciones.09.02.2016.01

Fuentes-Navarro, R. (2018a). Tendencias regionales y transnacionales de la investigación de la comunicación en América Latina. In D. Crovi \& R. Trejo (Coords.). Tejiendo nuestra historia. Investigación de la comunicación en América Latina (pp. 295-315). México: Unam.

Fuentes-Navarro, R. (2018b). Memoria e historicidad de la investigación en Comunicación en América Latina. In I. Coutinho \& A. Pereira (Orgs.). Intercom 40 anos: Comunicação, memórias e historicidades (pp. 17-28). São Paulo, SP: Intercom.

Fuentes-Navarro, R. (2018c). Concebir, enseñar, leer y escribir teorías de la comunicación, sus fundaciones y fundamentos. Revista Latinoamericana de Ciencias de la Comunicación, (28), 16-23.

Fuentes-Navarro, R. (2018d). La ciencia y la cultura como objetos de comunicación y práctica. In S. Herrera \& C. E. Orozco (Coords.). Comunicar ciencia en México. Prácticas y escenarios (pp. 15-42). Guadalajara: Iteso.

Garfield, E. (1955). Citation Indexes for Science, Science, 122( 3159), 108-111. doi: 10.1126/science.122.3159.108

Garfield, E. (1972). Citation Analysis as a tool in journal evaluation, Science, 178(4060), 471-479. doi: 10.1126/science.178.4060.471

Gehrke, P. J. \& Keith, W. M. (Eds.). (2015). A century of communication studies: the unfinished conversation. Londres; Nova York, NY: Routledge.

Günther, E. \& Domahidi, E. (2017). What communication scholars write about: an analysis of 80 years of research in high-impact journals. International Journal of Communication 11(2017), 3051-3071.

Herrera, K. et al. (Coord.) (2000). Una actualización biblio-hemerográfica de los estudios sobre comunicación en Bolivia 1990-2000. La Paz: Cibec.

Ioannidis, J. P. A. (2018). Meta-research: Why research on research matters. PLoS Biol, 16(3): e2005468. doi: 10.1371/journal.pbio.2005468

Ioannidis, J. P. A., Fanelli, D., Dunne D. D., \& Goodman, S. N. (2015). Metaresearch: Evaluation and Improvement of Research Methods and Practices. PLoS Biol, 13(10): e1002264. doi: 10.1371/journal.pbio.1002264 
Koivisto, J. \& Thomas, P. (2008). Mapping media and communication research: paradigms, institutions, challenges. Helsinki: Communication Research Center, University of Helsinki.

Kunsch, M. K. \& Denker, A. F. M. (Coords.). (1997). Produção científica brasileira em Comunicação. Década de 80, análises, tendências e perspectivas. São Paulo, SP: Intercom.

Levine, T. R. \& Hullett, C. R. (2015). Meta-Analysis. In W. Donsbach (Ed.). The Concise Encyclopedia of Communication (pp. 385-386). Malden, MA: Wiley-Blackwell.

Löblich, M. \& Scheu, A. M. (2011). Writing the History of Communication Studies: A Sociology of Science Approach, Communication Theory, 21(1), 1-22. doi: 10.1111/j.1468-2885.2010.01373.x

Löblich, M. \& Averbeck-Lietz, S. (2016). The transnational flow of ideas and histoire croisée with attention to the cases of France and Germany. In P. Simonson \& D. Park (Eds.), The International History of Communication Study (pp. 25-46). Nova York, NY; Londres, UK: Routledge.

Lopes, M. I. V. (Coord). (2012). Posgrados en Comunicación en Iberoamérica. Políticas Nacionales e Internacionales. São Paulo, SP: ECA-USP/Confibercom.

Lopes, M. I. V. (Org.). (2016). Epistemologia da comunicação no Brasil: Trajetórias autorreflexivas. São Paulo, SP: ECA-USP/AssIBERCOM.

Maldonado, E. (Coord). (2014). Panorâmica da investigação em comunicação no Brasil. Processos receptivos, cidadania e dimensão digital. Salamanca: Comunicación Social.

Marques de Melo, J. (Coord). (1984). Inventário da Pesquisa em Comunicação no Brasil 1883-1983. São Paulo, SP: Intercom; Alaic; CCID; CNPq.

Marques de Melo, J. (1988). Estudo comparativo dos sistemas de Comunicação Social no Brasil e no México, projeto de pesquisa. São Paulo, SP: Intercom/ Coneicc.

Marques de Melo, J. (Coord.). (1992). Comunicación Latinoamericana: Desafíos de la Investigación para el Siglo XXI. São Paulo, SP: ECA-USP/Alaic.

Marques de Melo, J. (1998). Teoria da Comunicação: paradigmas latino-americanos. Petrópolis, RJ: Vozes.

Marques de Melo, J. (2007). Entre el saber y el poder. Pensamiento comunicacional latino-americano. Monterrey: Comité Regional Norte de Cooperación con la Unesco.

Martín-Barbero, J. (1982). Retos a la investigación de comunicación en América Latina. Comunicación y Cultura, (9), 99-114.

Martín-Barbero, J. (1987). De los medios a las mediaciones. Comunicación, cultura y hegemonía. Barcelona: Gustavo Gili. 
Martín-Serrano, M. (2007). Teoría de la Comunicación. La comunicación, la vida y la sociedad. Madrid: McGraw Hill Interamericana.

Mattos, M. A., Barros, E. J. M., \& Oliveira, M. E. (Orgs.). (2018). Metapesquisa em Comunicação. O interacional e seu capital teórico nos textos da Compós. Porto Alegre, RS: Sulina.

Max Planck Gesselshaft (2003). Berlin Declaration on Open Access to Knowledge in the Sciences and Humanities. Recuperado de http://bit.ly/2IPAsV9

Merino, J. (1974). La investigación científica de la comunicación en América Latina. Chasqui, (5), 81-103.

Moragas, M. (2011). Interpretar la comunicación. Estudios sobre medios en América y en Europa. Barcelona: Gedisa.

Munafò, M. R. et al. (2017). A Manifesto for Reproducible Science. Nature Human Behavior 1, 0021(2017). doi: 10.1038/s41562-016-0021

Munizaga, G. \& Rivera, A. (1983). La investigación en Comunicación Social en Chile. Lima: Ceneca/Desco.

Nordenstreng, K (2007). Discipline or Field? Soul-searching in Communication Research. Nordicom Review, 28(Jubilee Issue), 211-222.

Orozco-Gómez, G. (1997). La investigación de la comunicación dentro y fuera de América Latina. Tendencias, perspectivas y desafíos del estudio de los medios. La Plata: Universidad Nacional de La Plata.

Pacchioni, G. (2018). The overproduction of truth. Passion, competition, and integrity in modern science. Oxford, UK: Oxford University Press.

Park, D. W. \& Pooley, J. (Eds.). (2008). The history of media and communication research: contested memories. Nova York, NY: Peter Lang.

Pasquali, A. (1978). Comprender la Comunicación. Caracas: Monte Ávila.

Peirano, L. \& Kudo, T. (1982). La investigación en comunicación social en el Perú. Lima: Desco.

Piñuel-Raigada, J. L. et al. (2013). Memoria Científico-Técnica del Proyecto MapCom: (El sistema de investigación en España sobre prácticas sociales de Comunicación. Mapa de Proyectos, Grupos, Líneas, Objetos de estudio y Métodos). Madrid: Programa Estatal de Fomento de la Investigación Científica y Técnica de Excelencia. Recuperado de https://bit.ly/2DCEUT2 Pisarek, W. (1982). COMNET: experience and perspective (Working Paper). Krakow: Cecom Unesco. Recuperado de https://unesdoc.unesco.org/ ark:/48223/pf0000052763

Pooley, J. D. \& Park, D. W. (2013). Communication Research. In P. Simonson, J. Peck, R. T. Craig, \& J. Jackson Jr. (Eds.). The Handbook of Communication History (pp. 76-90). Nova York, NY, Londres, UK: Routledge. 
Portillo, M. (Coord.) (2016). La investigación de la comunicación en México. Un panorama a través de las regiones a inicios del siglo XXI. México: Tintable/ AMIC.

Portugal, F. (Ed.) (2012). La investigación en comunicación social en América Latina 1970-2000 (Segunda edição, primera de 2000). Lima: Universidad Nacional Mayor de San Marcos.

Price, D. J. de S. (1963). Little Science, Big Science. Nova York, NY: Columbia University Press.

Rivera, J. B. (1986). La Investigación en Comunicación Social en Argentina. Lima: Asaicc; Desco/Alaic.

Rivera, J. B. (1997). Comunicación, medios y cultura. Líneas de investigación en la Argentina, 1986-1996. La Plata: Universidad Nacional de La Plata.

Simonson, P. \& Park, D. (Eds.). (2016). The International History of Communication Study. Nova York, NY; Londres, UK: Routledge.

Simonson, P. \& Peters, J. D. (2008). Communication and Media Studies, History to 1968. In W. Donsbach (Ed.). The International Encyclopedia of CommunicationI (Vol. 2, pp. 764-771). Nova York, NY: Blackwell.

Sodré, M. (2014). A ciência do comum. Notas para o método comunicacional. Petrópolis, RJ: Vozes.

Torrico, E. (2016). La Comunicación pensada desde América Latina (1960-2009). Salamanca: Comunicación Social.

Verón, E. (1987). La semiosis social. Fragmentos de una teoría de la discursividad. Barcelona: Gedisa.

Vizer, E. \& Vidales, C. (Coords.). (2016). Comunicación, campo(s), teorías y problemas. Una perspectiva internacional. Salamanca: Comunicación Social.

Waisbord, S. (2014). United and fragmented: Communication and media studies in Latin America, Journal of Latin American Communication Research, 4(1), 1-23.

Artigo recebido em 14 de abril e aprovado em 24 de abril de 2019. 